Relations industrielles

Industrial Relations

\title{
L'O.I.T. siège à Montréal
}

\section{The I.L.O's Conference at Montréal}

\section{Jean-Pierre Després}

Volume 2, numéro 1, 1946

URI : https://id.erudit.org/iderudit/1023674ar

DOI : https://doi.org/10.7202/1023674ar

Aller au sommaire du numéro

Éditeur(s)

Département des relations industrielles de l'Université Laval

ISSN

0034-379X (imprimé)

1703-8138 (numérique)

Découvrir la revue

Citer cet article

Després, J.P. (1946). L'O.I.T. siège à Montréal / The I.L.O's Conference at Montréal. Relations industrielles / Industrial Relations, 2(1), 1-3.

https://doi.org/10.7202/1023674ar

Tous droits réservés (C Département des relations industrielles de l’Université Laval, 1946
Ce document est protégé par la loi sur le droit d'auteur. L’utilisation des services d'Érudit (y compris la reproduction) est assujettie à sa politique d'utilisation que vous pouvez consulter en ligne.

https://apropos.erudit.org/fr/usagers/politique-dutilisation/ 


\section{Bulletin des Relations Industrielles}

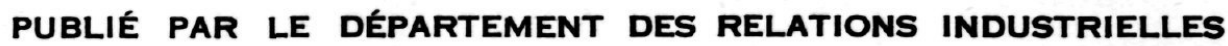

DE LA FACULTE DES SCIENCES SOCIALES DE LAVAL, QUÉBEC

T. R. P. G.-H. LÉVESQUE, o.p., doyen - GÉRARD TREMBLAY, directeur -- J. O'CONNELL-MAHER, assistant-directeur

GERARD DION, ptre, secrétaire - YVONNE B.-BERNARD, assistante-secrétaire

Dans ce numéro:

Réflexions sur les grèves $\ldots \ldots \ldots \ldots \ldots \ldots \ldots \ldots \ldots \ldots \ldots \ldots \ldots \ldots$

A propos de syndicalisme patronal. . . . . . .

\section{In this issue:}

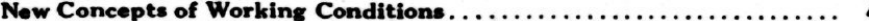

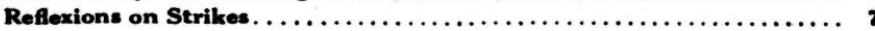

\section{L'O. I. T. \\ SIÈGE À MONTRÉAL}

\section{THE I. L. O's CONFERENCE AT MONTREAL}

Depuis le 19 septembre, la Conférence internationale du Travail tient sa $29^{\mathrm{e}}$ session à Montréal. C'est un événement que nous tenons à souligner en raison de l'importance grandissante de la réglementation internationale des conditions de travail et aussi à cause des relations très étroites qui existent entre le Bureau international du Travail et notre Département des Relations industrielles.

On sait que l'Organisation internationa!e du Travail a pour objet de faire régner la justice sociale dans le monde. Sa composition tripartite (groupes gouvernemental, patronal et ouvrier) explique le succès qu'elle a obtenu, depuis 1919, en dépit des ćchecs nombreux de la collaboration internationale entre les deux guerres. Le conflit qui vient de se terminer n'a pas enipêché l'O. I. T. de poursuivre son ouvre au cours de la tourmente. -Aujourd'hui, de toutes les institutions internationales spécialisées relićes à l'Organisation des Nations-Unies, l'Organisation internationale du Travail est celle qui possède le plus d'expérience, et son prestige est indiscutable. Les conventions et les recommandations adoptćes par la Conférence, depuis 1919, représentent une contribution unique au progrès social de l'humanité, particulièrem ent en ce qui concerne le relèvement des standards de travail. Bénéficiant d'une expérience précieuse, l'O.I.T. procède actuellement à la modification de sa constitution.en vue d'accrôttre son efficacití et son champ d'action. Isa ('onférence sera appelée à approuver un accord concernant la collahoration de l'O.I.T. au ('onseil ćconomigute et social des NationsUnies. ('est dire que la prósente session fera ćpoque dans les annales de l'Organisation internationale du Travail.

(Suite d la page \$)
Since the 19th of September the International Labour Conference has been holding its $29^{\text {th }}$ session at Montreal. This is an outstanding event about which we wish to say a few words on account of the increasing importance of the international regulation of working conditions and also on account of the connection which exists between the International Labour Office and our Departinent of Industrial Relations.

Needless to say that the purpose of the International Labour Organization is to further the reign of social justice throughout the world. Its tripartite constitution (governmental, capital and labour groups) enables one to understand why this organization has been so successful since 1919 despite numerous failures in international co-operation during the period intervening between the two great wars. The disastrous clash which has just come to an end did not prevent the I.L.O. from pursuing its worthy object throughout the turmoil, and to-day, of all the specialized international institutions carrying out work in connection with the programme of the United Nations Organization, the International Labour Organization is the most experienced and renowned. The agreements and recommendations passed by the Conference since 1919 constitute an unparalleled contribution to the social progress of humanity, most particularly as regards the raising of working standards. Guided by its vast experience the International Labour Office is actually nodifyng its constitution so as to increase its efficiency and widen its field of action. The Conference will be cal!ed upon to approve an agreement concerning the co-operation of the International Labour Office with the Fconomic and Social Council of the United Nations.

(Continued on page S) 


\section{RÉFLEXIONS SUR LES GRÈVES}

L'après-guerre est fertile en conflits du travail. Débutant aux Etats-Unis avec les grèves de l'automobile, du charbon et de l'acier, la plaie des différends industriels s'est propagée au Canada dans la plupart de nos industries nationales à une cadence telle que, d'après la statistique fédérale (Communiqué $n^{\circ} 1749$ du Ministère du Travail en date du 3 septembre 1946), les sept premiers mois de 1946 ont vu se déclarer 145 grèves intéressant 119,679 travailleurs et occasionnant une perte de $2,544,581$ journées individuelles de travail : ascension verticale par rapport à 1945 où la statistique n'a enregistré que 120 grèves affectant 37,609 travailleurs et causant une perte de seulement 128,208 journées de travail !

Si les grèves se continuent au même rythme d'ici la fin de la présente année, nous atteindrons au 31 décembre, en jours de travail perdus, le chiffre inégalé dans notre histoire ouvrière de 4,312,138. Ce serait dépasser de près d'un million l'année trouble de 1919 qui nous rappelle les grèves révolutionnaires d'inspiration bolchévique de l'Ouest canadien et du district de Winnipeg.

Le même phénomène s'était en effet produit à la fin de la guerre 1914-18. En 1918, il y eut 230 grèves affectant $\mathbf{7 9 , 7 4 3}$ travailleurs et leur faisant perdre 647,942 journées de travail. L'année suivante, 1919, connut 336 différends intéressant 148,915 ouvriers et occasionnant une perte de $3,400,942$ jours de travail.

Espérons que 1947, comme 1920, verra diminuer le nombre et l'importance des conflits. S'il est vrai que l'histoire se répète, voici ce que nous pou'vons escompter pour l'an prochain en faisant de la statistique comparative.

L'année 1920 a vu se dérouler 322 conflits intéressant 60,327 travailleurs et faisant perdre $\mathbf{7 1 9 , 5 2 4}$ jours ouvrables. Au cours de 1947 , si la statistique suit les mêmes courbes, nous enregistrerons une perte d'environ 900,000 journées de travail.

Les comparaisons ne riment souvent, dans ce domaine, qu'à peu de chose, mais il est toutefois des conclusions certaines à tirer en l'occurrence.

$1^{\circ}$ Les deux périodes d'après-guerre ont posé des problèmes de reconversion de l'industrie de guerre à l'industrie de paix et partant, de rajustements économiqures difficiles.

$\mathscr{2}^{\circ}$ Les conflits du travail ont été moins nombreux en 1946 qu'en 1919 mais ont eu plus d'importance individuelle. En effet, 145 grèves pour les sept premiers mois de 1946 à rapprocher de 336 grèves en 1919 , ou, sur une période de douze mois, probablement 250 grèves en 1946 contre 336 en 1919. Par contre, probablement 4,300,000 jours perdus en 1946 à rapprocher de $3,400,942$ en 1919. La durée moyenne par conflit a été, en 1919, de 10,122 jours; en 1946, elle sera probablement de 17,000 jours. Ouvriers et patrons sont done plus tenaces sur leurs positions aujourd'hui qu'il y a une génération.

$3^{\circ}$ La législation préventive des conflits de travail était rudimentaire en 1919 : elle se résumait aux lois de conciliation et d'arbitrage volontaire du Canada et des provinces. En 1946, au contraire, patrons et ouvriers ne peuvent légalement entrer en conflit sans recourir préalablement à la procédure de médiation. Si le salaire est l'objet du litige, l'arrêté C.P. 9384 oblige les parties à soumettre l'affaire au Conseil régional ou au Conseil national.

$4^{\circ}$ Les grèves de 1919 ont été déclarées dans la légalité, la loi ne les interdisant point et ne contraignant à aucune procédure particulière, sauf dans les utilités publiques. Au contraire, les grèves de 1946 ont été, pour la plupart, en violation flagrante des dispositions de l'arrêté C.P. 9384 relatif au contrôle des salaires. C'est, à proprement parler, le cas de la grève de l'automobile, de l'acier, du caoutchouc, des appäreils électriques et du textile. Il y a certes là un signe évident de l'affaiblissement du respect des lois et de l'autorité publique. Il faut signaler également que l'autorité constituée au Canada et dans toutes les provinces industrielles, sauf le Québec, n'a pas voulu recourir aux sanctions prévues par la loi ou les décrets-lois contre les fauteurs de grèves illégales.

Nous pourrions tirer d'autres conclusions sur les troubles industriels du temps présent. Qu'il suffise seulement de noter que le gouvernement fédéral s'inquiète de la situation et qu'il a décidé de convoquer, pour le 7 octobre prochain, une conférence des ministres provinciaux du Travail en vue d'étudier des propositions législatives qui remplaceront l'arrêté C.P. 9384 sur le contrôle des salaires et l'arrêté C.P. 1003 relatif aux relations industrielles. En d'autres termes, il s'agira de considérer un code national du travail. Nouvel effort de centralisation fédérale dans le domaine des questions du travail qui relèvent constitutionnellement de la compétence des provinces ! Il est d'ores et déjà certain que le Québec sera encore le champion de l'autonomie des provinces au cours des prochains pourparlers. Les attitudes sont déjà prises. Au reste, l'expérience fédérale en matière de travail n'a pas été très concluante. Les textes législatifs ont été excellents mais leur mise en vigueur généralem ent hésitantè, pour ne pas dire pire. L'autorité fédéra'e serait malvenue de s'ingérer dans des domaines où les nécessités de la guerre l'ont conduite, mais d'où elle doit se retirer maintenant que la paix est revenue. Flle peut tout au plus faciliter les échanges de vues entre les provinces afin d'amener celles-ci à suivre des normes aussi rapprochées que possible dans leur législation du travail. Du reste, cette standardisation est déjà très avancée et toutes les provinces industrielles ont génćralement des lois adéquates à leurs besoins. Il y a surtout une difficulté à résoudre : les bien mettre en application. C'est là une question de 
vigueur morale et d'énergie constructive dont, hélas ! la démocratie libérale manque très souvent.

$\mathrm{Au}$ premier plan demeure la question des salaires. Elle est à la source des grèves que nous déplorons et qui ralentissent la reprise de notre activité économique. Elle sera aussi le problème le plus épineux de la conférence du 7 octobre. Peut-on laisser devenir caduc l'arrêté C.P. 9384 sur le contrôle des salaires? Celuici, dans $90 \%$ des cas, a servi au relèvement des taux de salaires. Les Conseils régionaux qui l'appliquaient, par ordonnance souvent ou par autorisation, ont relevé de $40 \%$ en moyenne les salaires de 1941 . Ils ont prévenu de la sorte de multiples conflits. Les organisations ouvrières ont pris l'habitude de recourir à leurs bons offices qu'elles trouvent utiles malgré les critiques formulées.

L'économie du pays est-elle stabilisée au point que patrons et ouvriers puissent revenir à la liberté absolue de négociation sans danger de multiplier à l'infini les conflits de force? Si la digue pourtant bien faible du contrôle des salaires est brisée, pourra-t-on continuer le blocage des prix? Nous posons les problèmes sans les résoudre.

Une dernière considération sur la politique vitale des salaires. Il conviendrait que la prochaine conférence des ministres du Travail l'abordât sous un angle particulier. S'il est nécessaire, dans une industrie moderne, d'établir scientifiquement les taux gradués de rémunération de chaque classe de salariés, pourquoi l'autorité publique, sur le plan national, ne suggérerait-elle pas, après une enquête laborieuse conduite par des ingénieurs industriels, des taux de salaires moyens comparatifs pour la plupart des industries, commerces et services? Par exemple, le salaire moyen de l'ouvrier du textile, compte tenu des services rendus et des exigences de notre économie nationale, est-il équitable à \$0.60 l'heure? celui de l'ouvrier du bâtiment, à $\$ 1.00$ ? du métallurgiste, a $\$ 0.90$ ? Quelle est la valeur horaire relative du travail dans les diverses industries, eu égard aux facteurs de productivité, hygiène et sécurité, dextérité, régularité d'emploi, services rendus, etc.? L'enquête serait de vaste étendue et devrait être conduite à l'échelle nationale. Les normes suggérées à la suite des analyses du génie industriel seraient utiles aux employeurs comme aux salariés. Elles les guideraient dans leurs tractations et permettraient de corriger intelligemment les injustices actuelles. Pourquoi un secteur de l'économie serait-il plus malmené qu'un autre? Une opinion publique éclairée obligerait aux correctifs qui s'imposent. On objectera qu'il s'agit d'économie dirigée. Nous y sommes déjà enlisés, mais sous le signe du flou et de l'àpeu-près. A l'âge atomique, ne convient-il pas de caréner les relations entre les services d'un être humain et la récompense que lui doivent ses semblables? Et pour terminer sur le sujet qui a inspiré cet article, l'aménagement scientifique des taux de salaires dans une industrie et l'établissement de la valeur économique relative du travail dans les divers commerces, industries ou services ne réduiraient-ils pas à des minima insignifiants les conflits du travail?

Gerard Tremblay.

\section{RAPPORT DU CONGRES}

Le rapport du premier Congrès des Relations industrielles de Laval est maintenant prêt pour distribution. Le prix en est de $\$ 1.00$ et on peut l'obtenir en s'adressant à l'assistante-secrétaire du Département des Relations industrielles, Faculté des Sciences sociales. Université LavaI, Québec.

Ce rapport contient le texte des conférences qui furent prononcées au Château Frontenac, Je 11 mars dernier, et dont voici la liste :

a) L'Université et les relations industrielles ;

b) L'évolution des relations industrielles au Canada ;

c) L'apprentissage ;

d) Fatigue industrielle et productivité ;

e) Conciliation, arbitrage et tribunaux du travail ;

f) Les relations industrielles dans l'Etat moderne.

\section{L'O. I. T. SIEGE À MONTREAL}

(Suite de la page 1)

En plus de ces questions d'ordre constitutionnel, l'O.I.T prendra d'importantes décisions sur la protection des enfants et des jeunes travailleurs, surtout en ce qui a trait à l'examen médical d'aptitude à l'emploi et à la limitation du travail de nuit. Comme on le voit, les problèmes étudiés par la Conférence internationale du Travail sont de la plus grande actualité et les employeurs comme les travailleurs se doivent de suivre attentivement toutes les délibérations. Le mécanisme tripartite de l'O.I.T. permet aux organisations professionnelles patronales et ouvrières du Canada de participer directement à des travaux pré-législatifs ; cellesci dojvent donc s'intéresser tout particulièrement aux actıvités de l'Organisation internationale du Travail.

Jean-Pierre Despres.

\section{THE I. L. O's CONFERENCE AT MONTREAL}

\section{(From page 1)}

Therefore, the present session will mark an era in the history of the International Jabour Organization.

Besides dealing with matters pertaining to its constitution, the I.L.O. will come to certain important decisions regarding the protection of children and juvenile workers, specially as concerns a medical examination as to their fitness for employment and the limitation of night work. It is obvious that the problems studied by the International Labour Conference are questions of present interest; therefore, all employers and employees should closely follow the deliberations of the Conference. The very tripartite nature of the I.L.O. enables Canadian professional employers' and employees' organizations to take part directly in pre-legislative work, and for that reason, all those who are concerned with matters pertaining to labour should interest themselves in all the activites of the International Labour Organization. 